\title{
Diagnostic utility of cerebrospinal fluid immunocytochemistry for diagnosis of feline infectious peritonitis manifesting in the central nervous system
}

Journal of Feline Medicine and Surgery 2017, Vol. 19(6) 576-585 (C) The Author(s) 2016 Reprints and permissions: sagepub.co.uk/journalsPermissions.nav DOI: 10.1177/1098612X16640839 journals.sagepub.com/home/jfms

This paper was handled and processed by the European Editorial Office (ISFM) for publication in JFMS

\section{Stefanie Gruendl' ${ }^{1}$, Kaspar Matiasek ${ }^{2}$, Lara Matiasek ${ }^{1}$, Andrea Fischer ${ }^{1}$, Sandra Felten ${ }^{1}$, Konrad Jurina ${ }^{3}$ and Katrin Hartmann ${ }^{1}$}

\begin{abstract}
Objectives The aim of the study was to evaluate whether an ante-mortem diagnosis of central nervous system (CNS) feline infectious peritonitis (FIP) is possible via immunocytochemical staining (ICC) of feline coronavirus antigen (FCoV) within macrophages of cerebrospinal fluid (CSF).

Methods Prospectively, CSF samples of 41 cats were investigated, including cats with histopathologically confirmed FIP and neurological signs $(n=10)$, cats with confirmed FIP without CNS involvement $(n=11)$, cats with neurological signs but another confirmed CNS disease $(n=17)$, and cats without neurological signs and a disease other than FIP $(n=3)$. ICC staining of CSF macrophages was performed in all cats. Sensitivity, specificity, positive (PPV) and negative predictive values (NPV) of CSF ICC were calculated.

Results Of 10 samples from cats with CNS FIP, eight had detectable CSF macrophages, seven of which were positive for FCoV. Ten of 11 samples from cats with confirmed FIP without neurological signs had macrophages in the CSF, with all 10 being ICC-positive. In cats with other CNS disorders, 11/17 had macrophages, two of which stained positively. In cats with diseases other than FIP and without neurological disorders, 2/3 revealed macrophages, with one cat showing positive ICC staining. Diagnosis of FIP via CSF ICC had a sensitivity of $85.0 \%$ and a specificity of 83.3\%. PPV and NPV were $85.0 \%$ and $83.3 \%$.

Conclusions and relevance CSF ICC is a highly sensitive test for ante-mortem diagnosis of FIP manifesting in the CNS. However, CNS ICC specificity is too low to confirm FIP and the method should only be applied in conjunction with other features such as CSF cytology. CNS ICC could be helpful to discover pre-neurological stages of CNS FIP.
\end{abstract}

Accepted: 2 March 2016

\section{Introduction}

Feline infectious peritonitis (FIP) is a fatal immuneaugmented disease caused by feline coronaviruses (FCoV) that arises from mutation of the generally harmless enteric FCoV. ${ }^{1}$ This mutation allows the virus to infect and replicate in macrophages that carry the virus as a Trojan horse into remotely protected areas such as the eye and the central nervous system (CNS). ${ }^{2}$

The antibody prevalence of $\mathrm{FCoV}$ in cats ranges from $20 \%$ in single-cat households up to $100 \%$ in multi-cat households, but only approximately $5-10 \%$ of FCoVinfected cats develop FIP in multi-cat environments. ${ }^{3-12}$ Cats with FIP suffer from abdominal, pleural or pericardial effusion to a greater or lesser degree and/or granulomatous organ changes. ${ }^{8}$
${ }^{1}$ Clinic of Small Animal Medicine, Centre for Clinical Veterinary Medicine, Ludwig-Maximilians-Universitaet, Munich, Germany 2Section of Clinical and Comparative Neuropathology, Centre for Clinical Veterinary Medicine, Ludwig-Maximilians-Universitaet, Munich, Germany

${ }^{3}$ Small Animal Referral Centre, Haar, Germany

The results of this study were presented as an abstract at the 20th Eurocongress FECAVA in November 2014 and as an oral presentation at the 23rd annual InnLab (Innere Medizin und Labordiagnostik) conference in January 2015 in Leipzig.

\section{Corresponding author:}

Stefanie Gruendl, Clinic of Small Animal Medicine, Centre for Clinical Veterinary Medicine, Ludwig-Maximilians-Universitaet, Veterinaerstrasse 13, Munich, 80539 Munich, Germany Email: stefanie.gruendı@gmx.de 
If effusion is present, there are a number of diagnostic options; but, if there is no effusion, confirmation of the diagnosis requires histopathological confirmation of FIP via biopsy or post-mortem examination. In particular, the CNS form of FIP, which most commonly occurs without effusion, remains a post-mortem diagnosis in most cats. ${ }^{13}$ Pyogranulomatous meningoencephalitis and meningomyelitis lead to neurological signs in about $10 \%$ of cats affected by FIP. ${ }^{14-16}$ As the inflammatory lesions are predominantly surface-related, they typically lead to associated cerebrospinal fluid (CSF) changes. These include inflammatory pleocytosis with predominance of neutrophils and macrophages, and a markedly increased protein concentration. ${ }^{8,17,18}$ These findings are, however, not pathognomonic and can occur in a variety of infectious CNS diseases. ${ }^{19}$ More specifically, anti-coronavirus IgG can be detected in CSF.8,17 However, the presence of antibodies is also not diagnostic. ${ }^{20}$ In the study of Boettcher et $\mathrm{al}^{20}$ there was no significant difference in antibody titres in CSF from cats with neurological signs caused by FIP compared with cats with other neurological diseases. ${ }^{8}$ A recent promising approach was reported to demonstrate a $100 \%$ specificity in a prospective case-control study when identifying FCoV in CSF via real-time reverse transcriptase polymerase chain reaction (RT-qPCR). ${ }^{21}$ However, the sensitivity of this method was only $42.1 \%$ in all cats, and $85.7 \%$ in cats with neurological and/or ocular signs. Recently, a case report was published in which FCoV antigen in CSF macrophages was identified via immunocytochemical staining (ICC). ${ }^{22}$ This new method, however, has not been evaluated in controlled clinical trials.

Therefore, the aim of this study was to evaluate the diagnostic accuracy of ICC in a larger number of cats, including animals affected by CNS FIP and FIP without neurological signs, and those suffering from other diseases with and without neurological involvement.

\section{Materials and methods}

\section{Patients}

This study was designed as a prospective case-control study including 41 cats. Cats were divided into four groups: (1) cats with histologically confirmed CNS FIP $(\mathrm{n}=10)$; (2) cats with FIP without CNS involvement $(\mathrm{n}=11)$; (3) cats with neurological disorders caused by diseases other than FIP (CNS non-FIP, $\mathrm{n}=17$ ); (4) cats with diseases other than FIP without neurological signs (non-CNS non-FIP, $\mathrm{n}=3$ ). Cats had to fulfil the inclusion criteria shown in Table 1.

FIP diagnosis in cats with CNS FIP and cats with FIP without CNS involvement $(\mathrm{n}=21)$ was confirmed post mortem by histology, based on characteristic pyogranulomatous lesions, ${ }^{2,23}$ with immunohistochemistry (IHC) detecting intralesional evidence of macrophages positive for $\mathrm{FCoV}$ in affected organs, ${ }^{18,20,24,25}$ and exclusion of other pathogens on special stains (Table 1). In all cats with CNS FIP, immunohistochemically positive macrophages were detected within the CNS and, if affected, in other organs (Table 1). In cats with FIP without CNS involvement, immunopositive staining was demonstrated in at least one non-CNS organ (Table 1). Animals of the non-FIP groups $(n=20)$ had a survival time after onset of clinical signs of $>1.5$ years $(\mathrm{n}=9), 8,26,27$ or were euthanased and necropsied and did not show positive IHC staining for $\mathrm{FCoV}$ antigen in macrophages in any organs $(\mathrm{n}=11)$ (Table 1$)$.

CSF of neurological patients was collected for diagnostic reasons independent of the purpose of this study. The material was harvested by tapping of the cerebellomedullary cistern. In all other cats CSF was collected post mortem immediately upon euthanasia by either cisternal tap or transpallial puncture of the lateral ventricles.

\section{Immunocytochemistry}

Collected CSF was cytospun onto uncoated glass slides ( $\mathrm{R}$ Langenbrinck) immediately after collection using a cytocentrifuge (Hettich Zentrifugen, Universal 16R) according to standard protocols and stored at $-20^{\circ} \mathrm{C}$ $\left(-4^{\circ} \mathrm{F}\right)$ until further processing..$^{28,29}$

ICC was carried out manually using mouse monoclonal anti-coronavirus antibody (clone FIPV3-70; LINARIS $\mathrm{GmbH}$ ), an avidin-biotin complex detection kit (Vectastain; Vector Laboratory) and diaminobenzidine-tetrahydrochloride as chromagen. The staining protocol was based on standard guidelines for immunocytochemistry (ICC) ${ }^{30,31}$ In short, endogenous peroxidase activity was blocked by treatment with $0.7 \% \mathrm{H}_{2} \mathrm{O}_{2}$. The slides were then incubated with normal goat serum (dilution 1:20; MP Biomedicals) for 30 mins at room temperature after which they were coated with primary antibody (dilution 1:400) for $17 \mathrm{~h}$ at $4^{\circ} \mathrm{C}$ in a humid chamber, followed by labelling with biotinylated goat anti-mouse IgG (no. E 0433, dilution 1:200; DakoCytomation) and diaminobenzidine reaction. After immunolabelling, the slides were counterstained with Mayer's Haemalaun (AppliChem) and cover slips applied using a xylene-based mounting medium (Histokitt; Glaswarenfabrik Karl Hecht GmbH \& Co. KG).

For each specimen undergoing ICC, the presence and yield of immunopositive macrophages were assessed. Macrophages were assessed by scanning of the entire cytospin area, comprising 27 high-power fields at a magnification of at least $\times 400$ (Axiophot; Zeiss). Macrophage counts exceeding $>1$ macrophage per three fields of vision at $\times 200$ magnification were chosen as a cut-off for high cell yield.

\section{Cytology}

For microscopic evaluation of extracellular material, additional slides were stained with haematoxylin and eosin. CSF slides were evaluated by two independent raters blinded to the origin of the sample. Cytostained 


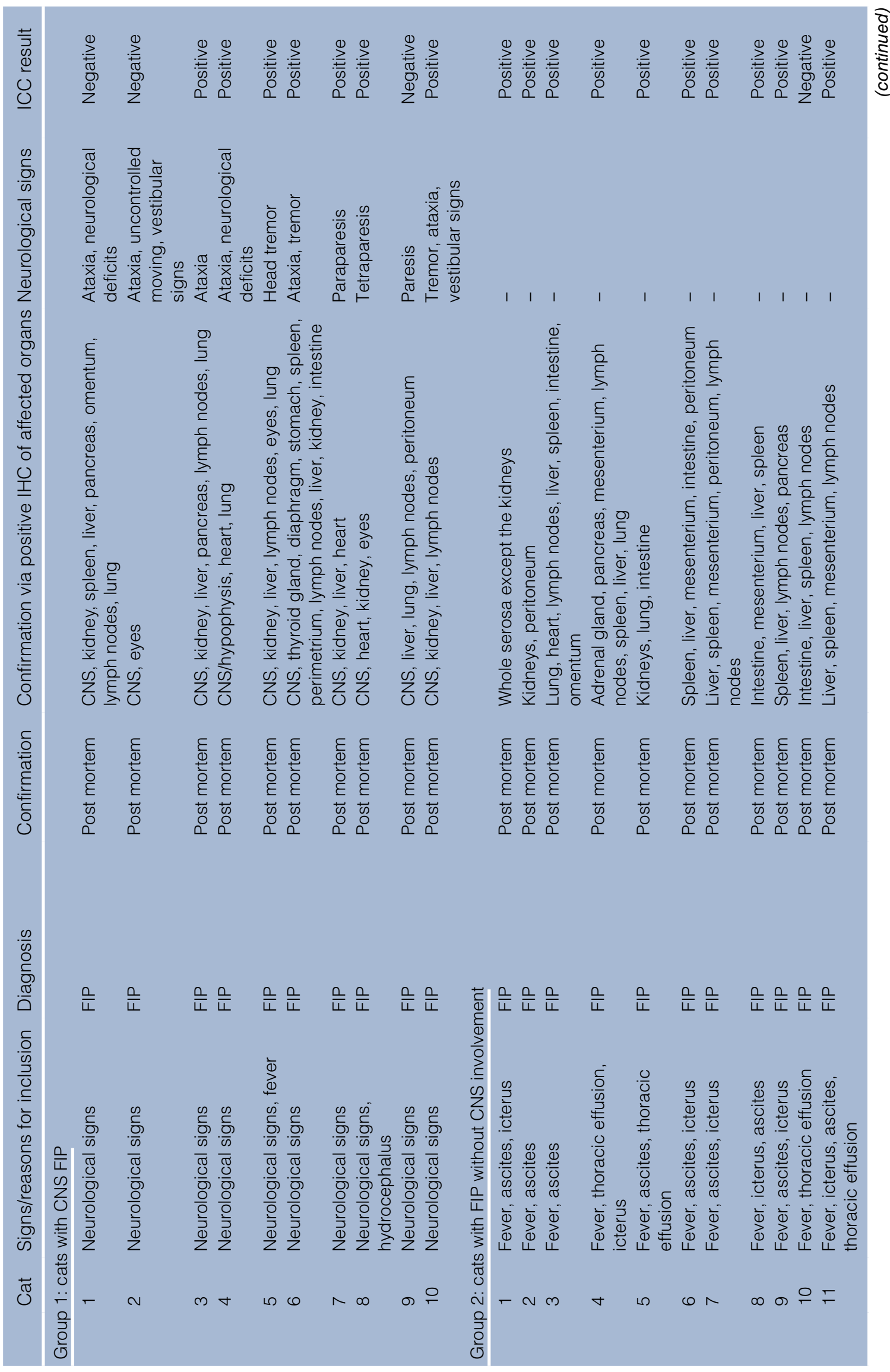




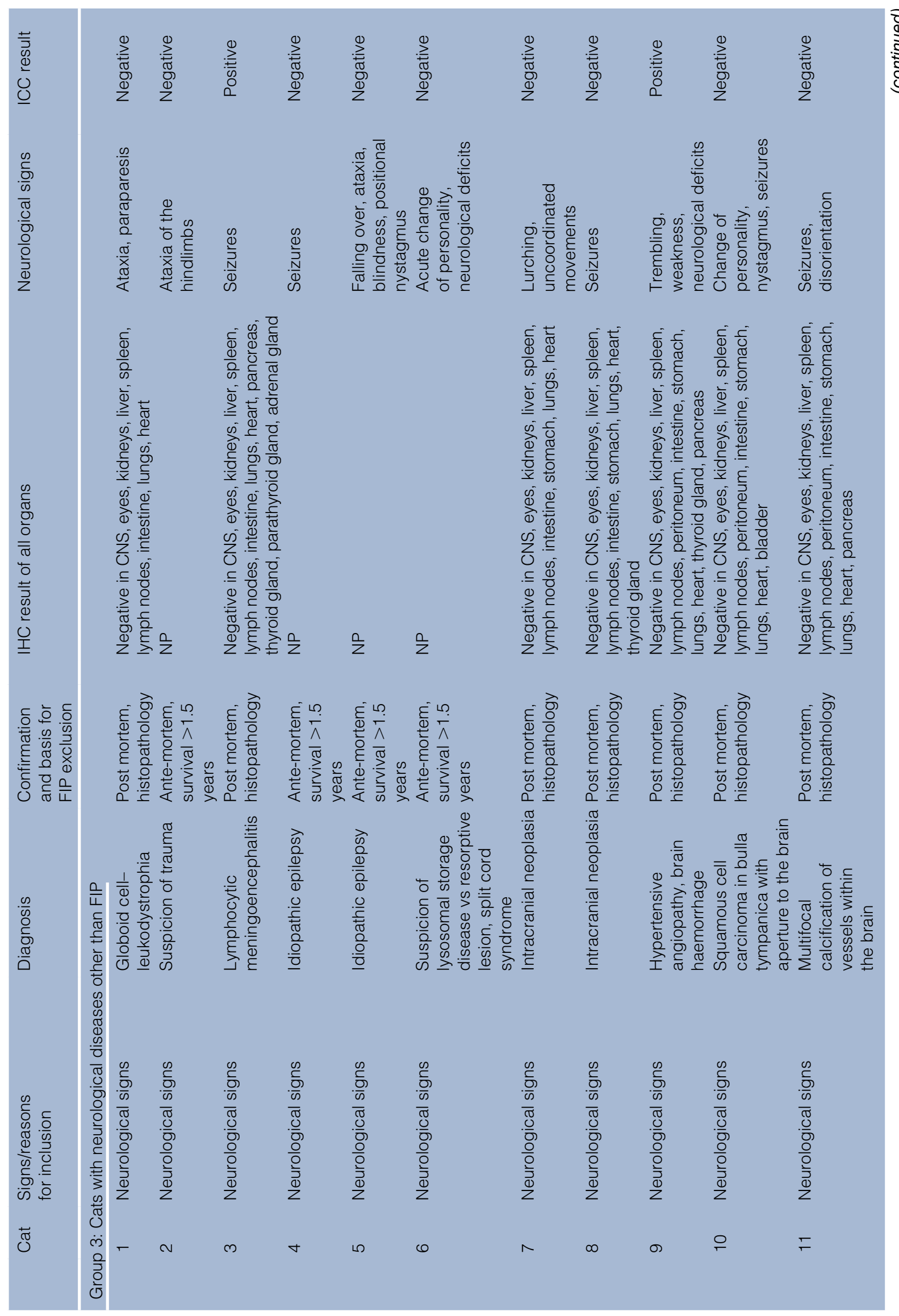




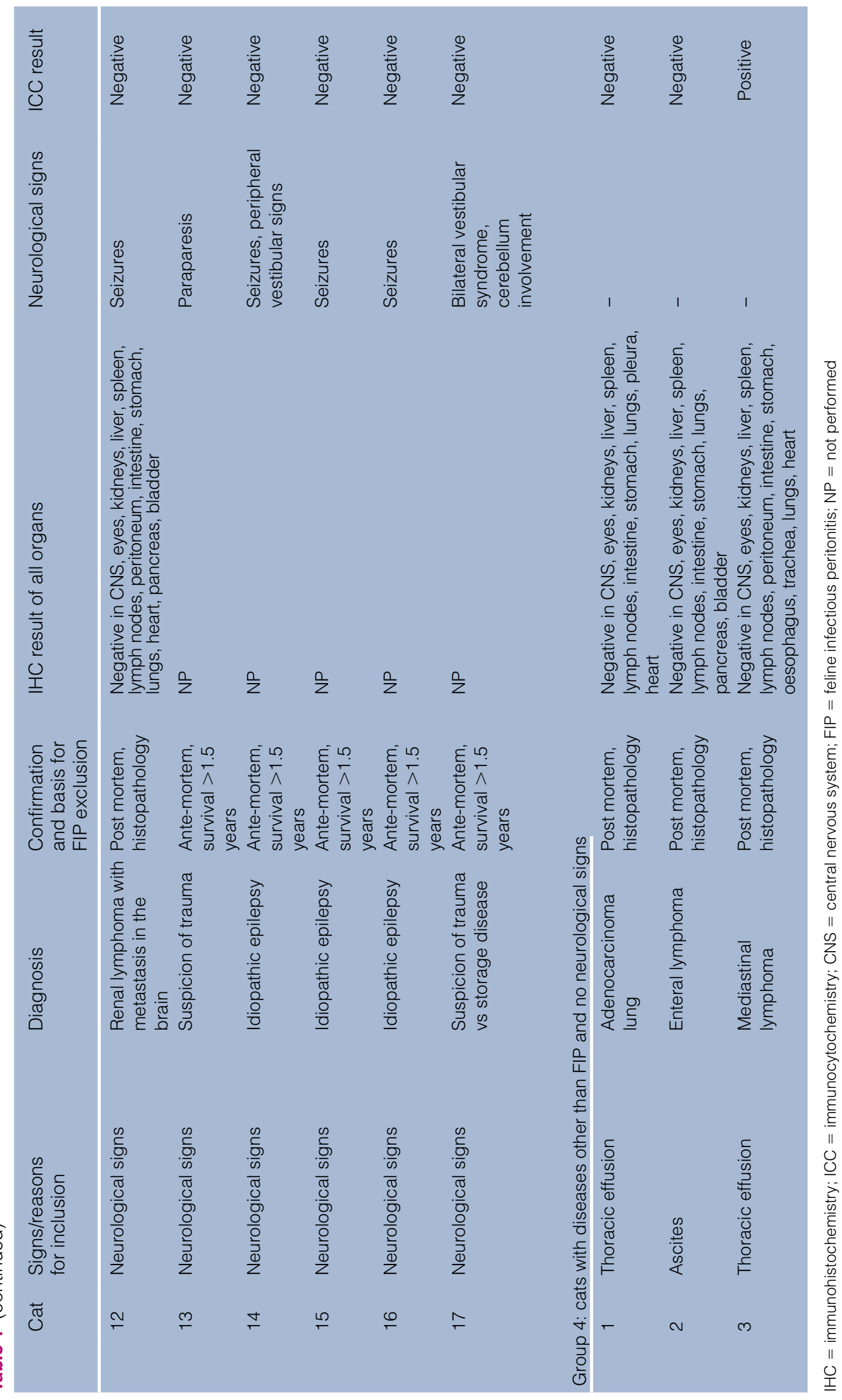




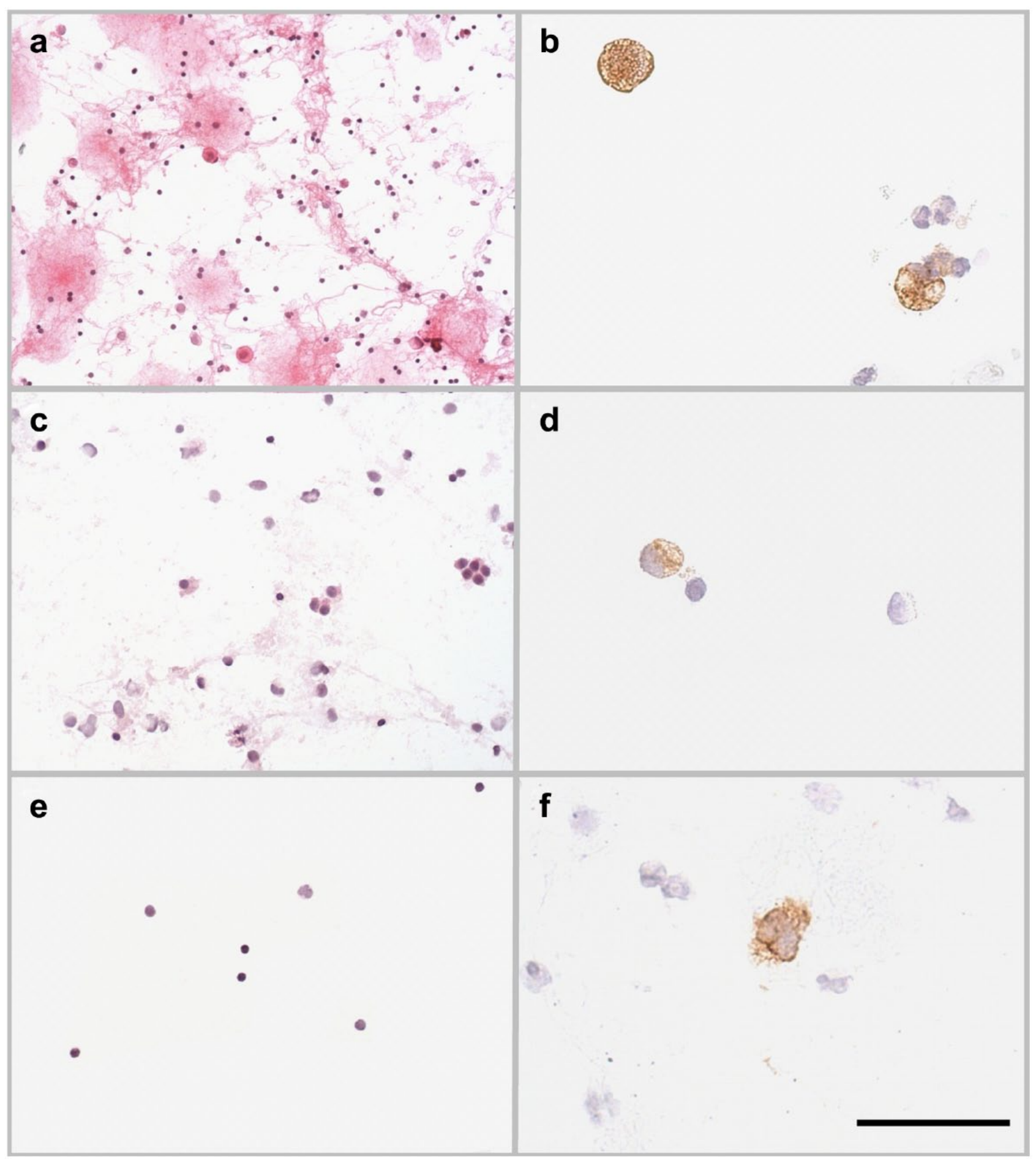

Figure 1 Cytological and immunocytochemical results. (a,c,e) Photomicrographs of slides indicative of feline infectious peritonitis (FIP) (a), compatible with FIP (c) and not indicative of FIP (e). (b,d,f) Immunopositive cerebrospinal fluid macrophages in confirmed central nervous system (CNS) FIP (b,d) and in a cat suffering from mediastinal lymphoma without CNS involvement $(f)$. Note that the staining intensity in the non-CNS non-FIP cat $(f)$ is similar to that of lower level expression in CNS FIP (d). Scale bars $=200 \mu \mathrm{m}(\mathrm{a}), 100 \mu \mathrm{m}(\mathrm{c}, \mathrm{e})$ and $50 \mu \mathrm{m}(\mathrm{b}, \mathrm{d}, \mathrm{f})$

slides were assessed for cellular content, composition and preservation, including evidence of bleeding, inflammation, microorganisms and brain tissue aspiration. Depending on the pattern of pleocytosis and protein content, the CSF was categorised as follows: (1) indicative of FIP if pyogranulomatous with macrophageal engulfment of neutrophils and absence of microorganisms and giant cells; (2) compatible with FIP in case of featuring mixed white blood cell population, including macrophages; and (3) not indicative of FIP owing to the lack of pleocytosis or showing other cellular composition such as exclusively neutrophilic cells (Figure 1). 


\section{Statistical evaluation}

The sensitivity and specificity, as well as the positive (PPV) and negative predictive values (NPV) were calculated for the whole group, as well as only for cats with neurological signs. Ninety-five percent confidence intervals were determined. Data analyses were performed using a two-sided Fisher's exact test with GraphPad Prism Version 5.0.

\section{Results}

CSF cytology

In the group of cats with CNS FIP, 9/10 CSF samples showed a significant pleocytosis. Eight of these nine cases were cytologically classified as indicative for FIP with pyogranulomatous inflammatory changes (Figure 1a). The sample of one cat did not contain cells. There was a mild blood content in two samples and a variable fibrin content in three samples (Table 2).

Ten of 11 samples of the cats with FIP without CNS involvement showed microscopic CSF abnormalities with the majority $(9 / 11)$ featuring inflammatory changes. FIPtypical pyogranulomatous reactions were seen in 4/11. A mild blood content was present in three samples and in two samples there was a mild or high fibrin content (Table 2).

In the cats with other diseases and neurological signs, $11 / 17$ samples showed inflammatory pleocytosis, 5/11 were unremarkable and $1 / 11$ samples did not contain cells. One sample with elevated cell counts was considered indicative of FIP. In one sample, taken via cisternal tapping, glioneuronal cells were observed. A mild-to-moderate blood content was seen in three samples and in four samples a mild-to-moderate fibrin content was visible (Table 2).

In the non-CNS non-FIP group, 2/3 samples had an abnormal CSF, while the third sample did not contain cells. The two abnormal slides showed inflammatory changes. One sample obtained via ventricle puncture contained glioneuronal cells. There was no blood or fibrin seen microscopically on the slides (Table 2).

\section{ICC}

In cats with CNS FIP, 8/10 samples contained macrophages. In seven of these, FCoV antigen was identified immunocytologically within the cytoplasm of the macrophages (Table 1; Figure 1b,d). In three of these cases, there was a high yield of immunopositive macrophages (Tables 3 and 4).

In cats with FIP without CNS involvement, macrophages were detected microscopically in 10/11 CSF samples, all of which stained positive for FCoV antigen (Table 1). The high-to-low yield ratio among these samples was $8: 2$, which corresponded to the overall high nucleated cellularity (Tables $2-4$ ).

In the cats with other diseases and with neurological signs, macrophages were present in 11 samples. In two of these samples, the macrophages stained positive for $\mathrm{FCoV}$ antigen with one showing a high yield (Tables 1, 3 and 4).

In the cats with other diseases and without neurological signs, 2/3 samples showed macrophages with one showing a positive ICC staining with high yield (Tables 1,3 and 4, Figure 1f).

\section{Discussion}

Intra-vitam diagnosis of FIP in cats with neurological signs that lack body cavity effusions has always been a challenge. $4,8,32$ Owing to the meningeal involvement of CNS manifestations of FIP, most efforts have concentrated on CSF analysis in order to establish a valuable diagnostic test. ${ }^{18,20-22}$ Cytological changes, even though effectively narrowing differentials, are non-specific. Moreover, depending on the location of the pyogranulomatous reaction, vascular compromise and concurrent lymphoproliferative changes, the degree and composition of pleocytosis can vary and might not be indicative for FIP or even mimic other diseases like CNS lymphoma..$^{24,33}$

Hence, the purpose of this study was to evaluate whether an ante-mortem diagnosis of CNS FIP is possible via ICC staining of FCoV antigen within macrophages of the CSF. ${ }^{21}$ It was demonstrated that FIP could be correctly diagnosed via ICC staining of the CSF in $81.0 \%$ of the cats with immunohistochemically confirmed FIP. Thus, the ICC of the CSF had a sensitivity of $85.0 \%$ and a PPV of $85.0 \%$. Interestingly, the sensitivity of the test was lower in cats with FIP with CNS involvement (77.8\%) than in those with FIP without CNS involvement (91.0\%) (Table 4 ). The sensitivity of $77.8 \%$ of ICC in cats with CNS FIP was relatively low, when considering that immunopositive macrophages were detected within the CSF in as much as $90.9 \%$ of cats with FIP but without histological evidence of CNS involvement. As only three of these cats exhibited very mild blood contamination, this phenomenon cannot be attributed entirely to a spillover of infected macrophages. Instead, a majority of ICC-positive animals showed inflammatory pleocytosis, of which 4/9 were FIP-indicative. Even though less likely due to the high cellularity of the CSF, the pure presence of infected monocytes and macrophages, accompanied by lymphocytes, can resemble a bystander effect of systemic inflammation. ${ }^{34-38}$ It is more likely that circulating macrophages have been recruited to the CNS owing to local inflammation. This can happen during immune complex deposition, which can occur as an initial step of FIP manifestation of the brain preceding neurological signs. In this study, none of the cats with FIP without CNS involvement had macroscopic or histological lesions in the CNS. Therefore, these cats with FIP without CNS involvement could have been at the beginning of neurological manifestation of the disease, still without clinical signs and pathological lesions. ${ }^{21}$ In a study of Doenges et al, ${ }^{21}$ 2/12 cats with FIP without neurological/ocular signs showed also a positive RT-qPCR result. In one of these cats, the histological examination of the brain revealed inflammatory changes and necrotic lesions within the CNS. The other cat had no gross or histological lesions. ${ }^{21}$ Hence, in these two cats with FIP without neurological/ocular signs, RT-qPCR detected 
Table 2 Microscopic evaluation and immunocytochemistry (ICC) results

\begin{tabular}{|c|c|c|c|c|c|c|c|c|}
\hline \multirow[b]{2}{*}{ ICC result } & \multicolumn{2}{|c|}{ CNS FIP $(n=10)$} & \multicolumn{2}{|c|}{$\begin{array}{l}\text { FIP without CNS } \\
\text { involvement }(n=11)\end{array}$} & \multicolumn{2}{|c|}{$\begin{array}{l}\text { CNS non-FIP } \\
(\mathrm{n}=17)\end{array}$} & \multicolumn{2}{|c|}{$\begin{array}{l}\text { Non-CNS non-FIP } \\
(n=3)\end{array}$} \\
\hline & $\begin{array}{l}\text { ICC } \\
\text { positive } \\
(n=7)\end{array}$ & $\begin{array}{l}\text { ICC } \\
\text { negative } \\
(n=3)\end{array}$ & $\begin{array}{l}\text { ICC } \\
\text { positive } \\
(n=10)\end{array}$ & $\begin{array}{l}\text { ICC } \\
\text { negative } \\
(n=1)\end{array}$ & $\begin{array}{l}\text { ICC } \\
\text { positive } \\
(n=2)\end{array}$ & $\begin{array}{l}\text { ICC } \\
\text { negative } \\
(n=15)\end{array}$ & $\begin{array}{l}\text { ICC } \\
\text { positive } \\
(n=1)\end{array}$ & $\begin{array}{l}\text { ICC } \\
\text { negative } \\
(n=2)\end{array}$ \\
\hline \multicolumn{9}{|l|}{ Pleocytosis } \\
\hline None & 0 & 0 & 1 & 1 & 0 & 5 & 0 & 0 \\
\hline $\mathrm{PgP}$ & 7 & 1 & 4 & 0 & 0 & 1 & 1 & 0 \\
\hline $\mathrm{LmP}$ & 0 & 1 & 3 & 0 & 1 & 4 & 0 & 1 \\
\hline $\mathrm{MP}$ & 0 & 0 & 1 & 0 & 0 & 1 & 0 & 0 \\
\hline MMP & 0 & 0 & 1 & 0 & 0 & 0 & 0 & 0 \\
\hline MLmP & 0 & 0 & 0 & 0 & 0 & 2 & 0 & 0 \\
\hline MLNP & 0 & 0 & 0 & 0 & 1 & 0 & 0 & 0 \\
\hline UP & 0 & 0 & 0 & 0 & 0 & 1 & 0 & 0 \\
\hline No cells visible & 0 & 1 & 0 & 0 & 0 & 1 & 0 & 1 \\
\hline \multicolumn{9}{|l|}{ Nucleated cell content } \\
\hline Low & 3 & 1 & 2 & 1 & 1 & 12 & 0 & 1 \\
\hline High & 4 & 2 & 8 & 0 & 1 & 3 & 1 & 1 \\
\hline \multicolumn{9}{|l|}{ Blood content* } \\
\hline- & 5 & 3 & 7 & 1 & 2 & 12 & 1 & 2 \\
\hline+ & 2 & 0 & 3 & 0 & 0 & 1 & 0 & 0 \\
\hline++ & 0 & 0 & 0 & 0 & 0 & 2 & 0 & 0 \\
\hline+++ & 0 & 0 & 0 & 0 & 0 & 0 & 0 & 0 \\
\hline \multicolumn{9}{|l|}{ Fibrin content* } \\
\hline- & 5 & 2 & 7 & 1 & 1 & 11 & 1 & 2 \\
\hline+ & 1 & 0 & 1 & 0 & 0 & 2 & 0 & 0 \\
\hline++ & 1 & 1 & 0 & 0 & 1 & 2 & 0 & 0 \\
\hline+++ & 0 & 0 & 1 & 0 & 0 & 0 & 0 & 0 \\
\hline Unclear & 0 & 0 & 1 & 0 & 0 & 0 & 0 & 0 \\
\hline Indicative of FIP & 7 & 1 & 4 & 0 & 0 & 1 & 1 & 0 \\
\hline FIP-compatible & 0 & 0 & 4 & 0 & 1 & 2 & 0 & 1 \\
\hline Not indicative of FIP & 0 & 1 & 2 & 1 & 1 & 11 & 0 & 0 \\
\hline Inflammatory & 0 & 1 & 0 & 0 & 1 & 8 & 0 & 0 \\
\hline Non-inflammatory & 0 & 0 & 2 & 1 & 0 & 3 & 0 & 0 \\
\hline
\end{tabular}

*(-) indicates no blood or fibrin content; $(+++)$ indicates highest blood/fibrin content

$\mathrm{CNS}=$ central nervous system; FIP = feline infectious peritonitis; PgP = pyogranulomatous pleocytosis; $\mathrm{LmP}=$ lymphomonocytic pleocytosis; $\mathrm{MP}=$ monocytic pleocytosis; $\mathrm{MMP}=$ mixed, predominantly monocytic pleocytosis; MLmP = mixed, predominantly lymphomonocytic pleocytosis; MLNP = mixed lymphoid and neutrophilic pleocytosis; UP = unclear pleocytosis

Table 3 Cell yield of immunocytochemical (ICC)-positive samples

\begin{tabular}{|lllll} 
& CNS FIP & FIP without CNS involvement & CNS non-FIP & Non-CNS non-FIP \\
\hline Positive ICC results & 7 & 10 & 2 & 1 \\
High yield & 3 & 8 & 1 & 1 \\
Low yield & 4 & 2 & 1 & 0
\end{tabular}

CNS = central nervous system; FIP = feline infectious peritonitis

the FCoV within the CSF. Owing to the low sensitivity of the RT-qPCR (42.1\%), the other CSF samples of cats with FIP might have been false negative in this study. ${ }^{21}$ Comparing PCR results vs ICC results regarding FIP diagnosis on a same patient population should be investigated in further studies.
Unfortunately, specificity (83.3\%) and the NPV (83.3\%) of ICC were not as high as expected. Three samples (15.0\%) of the control groups contained immunopositive macrophages. This finding severely compromises the validity of the ICC. Independent of the presence (two cats) or absence (one cat) of neurological signs and brain changes 
Table 4 Sensitivity and specificity of cerebrospinal fluid immunocytochemistry in cats with feline infectious peritonitis (FIP)

\begin{tabular}{|c|c|c|c|}
\hline & All cats & Cats with neurological signs & Cats without neurological signs \\
\hline Sensitivity & $85.0(\mathrm{Cl} 62.1-96.8)$ & $77.8(\mathrm{Cl} 40.0-97.1)$ & $91.0(\mathrm{Cl}$ 58.7-99.8) \\
\hline Specificity & $83.3(\mathrm{Cl} 58.6-96.4)$ & $87.5(\mathrm{Cl} 61.7-98.5)$ & $50.0(\mathrm{Cl} 1.3-98.7)$ \\
\hline PPV & $85.0(\mathrm{Cl} 62.1-96.8)$ & $77.8(\mathrm{Cl} 40.0-97.1)$ & $90.1(\mathrm{Cl} 58.7-99.8)$ \\
\hline NPV & $83.3(\mathrm{Cl} 58.6-96.4)$ & $87.5(\mathrm{Cl} 61.7-98.5)$ & $50.0(\mathrm{Cl} 1.3-98.7)$ \\
\hline Prevalence of FIP & $52.6(\mathrm{Cl} 35.8-69.0)$ & $36.0(\mathrm{Cl} 18.0-57.5)$ & $85.0(\mathrm{Cl} 54.6-90.1)$ \\
\hline
\end{tabular}

Data are \% (95\% confidence interval)

PPV $=$ positive predictive value; NPV = negative predictive value

(two cats), all these false-positive CSF samples showed inflammatory features that were pyogranulomatous, lymphoid and neutrophilic or lymphomonocytic (Tables 1 and 2 ). Hence, the cytopathological pattern of the one cat with a mediastinal lymphoma and without neurological signs would be compatible with a preliminary stage of FIP. The cytopathological pattern of the other two cats with neurological signs would also be compatible with either a preliminary stage of FIP or, alternatively, with homing of FCoV-infected macrophages to the CNS in the course of other inflammatory CNS disorders. Thus, it is possible in all three cases that the cats actually suffered from early FIP and other diseases simultaneously. However, immunohistochemical investigations of these cats were negative, making this possibility rather unlikely.

Another reason for the false-positive results is that the immunocytological assay cannot distinguish between mutated FCoV (FIPV) and non-virulent FCoV (FECV) that also is able to infect and replicate in macrophages to some extent. ${ }^{39}$ It has been documented that productive and sustainable virus replication in macrophages only occurs after mutation of $\mathrm{FCoV}, 2$ while the general ability of non-virulent FCoV (FECV) to infect macrophages is limited and goes with a low staining signal. ${ }^{39}$ Thus, a positive staining of non-mutated virus within macrophages is unlikely.

Non-specific staining and aberrant antibody binding have to also be considered as reasons for the false-positive staining results. Endogenous peroxidase activity has been effectively quenched by pretreatment with $\mathrm{H}_{2} \mathrm{O}_{2}$. The chosen monoclonal primary antibody has been used in multiple studies on FIP and is directed at coronavirus nucleocapsids..$^{25,26,40}$ It is known to react with FCoV serotypes 1 and 2, ferret coronavirus, canine coronavirus, transmissible gastroenteritis virus and bovine coronavirus. ${ }^{41}$ However, there are no reports on affinity to endogenous epitopes and structures, so that non-specific staining is unlikely but possible.

There are some limitations in the study. First of all, only a low amount of CSF could be collected in most cats with FIP. FIP is a disease affecting mostly young cats, $<1$ years of age, that usually have a body weight $<2 \mathrm{~kg}$. Hence, the amount of CSF that could be collected was $<0.5 \mathrm{ml}$ (when following the guidelines to take not more than $1 \mathrm{ml} / 5 \mathrm{~kg}^{42}$ ). Accordingly, only a few cells could be gained from the samples. Although CSF was immediately preserved, the instability of CSF cells might be damaged by freezing or washed off during immunolabelling. Thus, often only a few cells were available for interpretation of the ICC, especially in cases with no inflammatory CSF. Another limitation is that FIP could not be excluded for sure in the control cats that could have had FIP beside another underlying disease, although IHC of all organs was negative.

\section{Conclusions}

ICC on CSF taps was shown to be a sensitive test for diagnosis of FIP, regardless of whether the CNS was involved or not. Unfortunately, the specificity of the method was not high enough. Immunopositivity of CSF macrophages in cats with neurological signs, but without detectable FIP on post-mortem examination, might precede histological changes of FIP. When summarising the results of this study, ICC of CSF cannot be considered a useful test for confirmation of FIP.

Acknowledgements We are grateful to Mrs Karin Stingl for excellent technical assistance.

Conflict of interest The authors declared no potential conflicts of interest with respect to the research, authorship, and/ or publication of this article.

Funding The authors received no financial support for the research, authorship, and/or publication of this article.

\section{References}

1 Pedersen NC. Coronavirus diseases (coronavirus enteritis, feline infectious peritonitis). In: Holzworth J (ed). Diseases of the cat: medicine and surgery. Philadelphia, PA: WB Saunders Company, 1987, pp 193-214.

2 Kipar A and Meli ML. Feline infectious peritonitis: still an enigma? Vet Pathol 2014; 51: 505-526.

3 Brown MA, Troyer JL, Pecon-Slattery J, et al. Genetics and pathogenesis of feline infectious peritonitis virus. Emerg Infect Dis 2009; 15: 1445-1452.

4 Pedersen NC. A review of feline infectious peritonitis virus infection: 1963-2008. J Feline Med Surg 2009; 11: 225-258. 
5 Addie DD. Clustering of feline coronaviruses in multicat households. Vet J 2000; 159: 8-9.

6 Addie DD and Jarrett O. A study of naturally occurring feline coronavirus infections in kittens. Vet Rec 1992; 130: 133-137.

7 Kennedy M, Citino S, McNabb AH, et al. Detection of feline coronavirus in captive Felidae in the USA. $J$ Vet Diagn Invest 2002; 14: 520-522.

8 Hartmann K. Feline infectious peritonitis. Vet Clin North Am Small Anim Pract 2005; 35: 39-79.

9 Pedersen NC. Serologic studies of naturally occurring feline infectious peritonitis. Am J Vet Res 1976; 37: 1449-1453.

10 Pedersen NC. Feline infectious peritonitis: something old, something new. Feline Pract 1976; 6: 42-51.

11 Addie D and Jarrett $\mathrm{O}$. Feline coronavirus antibodies in cats. Vet Res 1992; 131: 202-203.

12 Addie D, Toth S, Murray GD, et al. Risk of feline infectious peritonitis in cats naturally infected with feline coronavirus. Am J Vet Res 1995; 56: 429-434.

13 Norris JM, Bosward KL, White JD, et al. Clinicopathological findings associated with feline infectious peritonitis in Sydney, Australia: 42 cases (1990-2002). Aust Vet J 2005; 83: 666-673.

14 Rohrer C, Suter PF and Lutz H. The diagnosic of feline infectious peritonitis (FIP) - retrospective and prospective study. Kleintierpraxis 1993; 38: 379-389.

15 Kline KL, Joseph RJ and Averill DR. Feline infectious peritonitis with neurological involvement: clinical and pathological findings in 24 cats. J Am Anim Hosp Assoc 1994; 30: 111-118.

16 Slauson DO and Finn JP. Meningoencephalitis and panophthalmitis in feline infectious peritonitis. $J$ Am Vet Med Assoc 1972; 160: 729-734.

17 Foley JE, Lapointe JM, Koblik P, et al. Diagnostic features of clinical neurologic feline infectious peritonitis. $J$ Vet Intern Med 1998; 12: 415-423.

18 Steinberg TA, Boettcher IC, Matiasek K, et al. Use of albumin quotient and IgG index to differentiate blood- vs brain-derived proteins in the cerebrospinal fluid of cats with feline infectious peritonitis. Vet Clin Pathol 2008; 37: 207-216.

19 Gunn-Moore DA and Reed N. CNS disease in the cat: current knowledge of infectious causes. J Feline Med Surg 2011; 13: 824-836.

20 Boettcher IC, Steinberg T, Matiasek K, et al. Use of anti-coronavirus antibody testing of cerebrospinal fluid for diagnosis of feline infectious peritonitis involving the central nervous system in cats. J Am Vet Med Assoc 2007; 230: 199-205.

21 Doenges SJ, Weber K, Dorsch R, et al. Detection of feline coronavirus in cerebrospinal fluid for diagnosis of feline infectious peritonitis in cats with and without neurological signs. J Feline Med Surg 2016; 18: 104-109.

22 Ives EJ, Vanhaesebrouck AE and Cian F. Immunocytochemical demonstration of feline infectious peritonitis virus within cerebrospinal fluid macrophages. J Feline Med Surg 2013; 15: 1149-1153.

23 Dahme E and Weiss E. Grundriss der speziellen pathologischen Anatomie der Haustiere. 6th ed. Stuttgart: Enke Verlag, 2007.

24 Kipar A, Bellmann S, Kremendahl J, et al. Cellular composition, coronavirus antigen expression and production of specific antibodies in lesions in feline infectious peritonitis. Vet Immunol Immunopathol 1998; 65: 243-257.
25 Tammer R, Evensen O, Lutz H, et al. Immunohistological demonstration of feline infectious peritonitis virus antigen in paraffin-embedded tissues using feline ascites or murine monoclonal antibodies. Vet Immunol Immunopathol 1995; 49: 177-182.

26 Pedersen NC. An overview of feline enteric coronavirus and feline infectious peritonitis virus infection. Feline Pract 1995; 23: 7-20.

27 Robison RL, Holzworth J and Gilmore CE. Naturally occurring feline infectious peritonitis: signs and clinical diagnosis. J Am Vet Med Assoc 1971; 158 Suppl 2: 981-986.

28 Fry MM, Vernau W, Kass PH, et al. Effects of time, initial composition, and stabilizing agents on the results of canine cerebrospinal fluid analysis. Vet Clin Pathol 2006; 35: 72-77.

29 Storch-Hagenlocher B, Reiber H, Wildemann B, et al. Liquordiagnostik. In: Wildemann B, Oschmann $\mathrm{P}$ and Reiber $\mathrm{H}$ (eds). Neurologische Labordiagnostik. Stuttgart, New York: Georg Thieme Verlag KG, 2006, pp 30-73.

30 Ramos-Vara JA and Miller MA. When tissue antigens and antibodies get along: revisiting the technical aspects of immunohistochemistry - the red, brown, and blue technique. Vet Pathol 2014; 51: 42-87.

31 Dako, Boenisch T, Taylor CR, et al. Education guide/ immunohistochemical (IHC) staining methods. 5th ed. Carpinteria, CA: Dako North America, 2009, p 172.

32 Kipar A, Koehler K, Bellmann S, et al. Feline infectious peritonitis presenting as a tumour in the abdominal cavity. Vet Rec 1999; 144: 118-122.

33 Singh M, Foster DJ, Child G, et al. Inflammatory cerebrospinal fluid analysis in cats: clinical diagnosis and outcome. J Feline Med Surg 2005; 7: 77-93.

34 Webb AA and Muir GD. The blood-brain barrier and its role in inflammation. J Vet Intern Med 2000; 14: 399-411.

35 Engelhardt B and Wolburg H. Mini-review: transendothelial migration of leukocytes: through the front door or around the side of the house? Eur J Immunol 2004; 34: 2955-2963.

36 Wolburg H, Wolburg-Buchholz K and Engelhardt B. Diapedesis of mononuclear cells across cerebral venules during experimental autoimmune encephalomyelitis leaves tight junctions intact. Acta Neuropathol 2005; 109: 181-190.

37 Konsman JP, Drukarch B and Van Dam AM. (Peri)vascular production and action of pro-inflammatory cytokines in brain pathology. Clin Sci (Lond) 2007; 112: 1-25.

38 Bolton SJ, Anthony DC and Perry VH. Loss of the tight junction proteins occludin and zonula occludens-1 from cerebral vascular endothelium during neutrophil-induced blood-brain barrier breakdown in vivo. Neuroscience 1998; 86: $1245-1257$.

39 Poland AM, Vennema H, Foley JE, et al. Two related strains of feline infectious peritonitis virus isolated from immunocompromised cats infected with a feline enteric coronavirus. J Clin Microbiol 1996; 34: 3180-3184.

40 Hok K. Demonstration of feline infectious peritonitis virus in conjunctival epithelial cells from cats. A simple and reliable method for clinical veterinary virology screening. APMIS 1989; 97: 820-824.

41 Linaris. Mouse anti-feline coronavirus monoclonal. http: / / www.linaris.de/datenblatt/DBL-E-MAK2194.pdf (2015, accessed 11 March 2016).

42 Dewey CW and Ducoté JM. Neurodiagnostics. In: Dewey CW (ed). A practical guide to canine and feline neurology. 2nd ed. Ames, IA: Wiley-Blackwell, 2008, pp 75-76. 